\title{
Turismo Espacial: Cuestiones legales que afectan a este tipo de turismo
}

\section{Spatial Tourism: Legal issues that concern this type of tourism}

\author{
Ana Isabel Serrano Navalón (SERRANO NAVALÓN, A. I.) \\ Francisca Ramón Fernández (RAMÓN FERNÁNDEZ, F.) ${ }^{* *}$
}

RESUMEN - El objetivo principal de este trabajo es analizar las principales cuestiones legales que afectan al turismo espacial, incidiendo en la principal legislación vigente aplicable. La metodología utilizada en la elaboración del presente trabajo ha partido del estudio de la definición de este tipo de turismo y su diferenciación con otras tipologías, y los resultados obtenidos permitirán considerar la viabilidad de creación de hoteles para acoger a los turistas, teniendo en cuenta su peculiaridad y la potencialidad turística.

Palabras clave: Turismo; Espacio; Derecho; Astronauta.

\begin{abstract}
This paper aims to analyze the main legal issues that concern the spatial tourism, focusing in the main applicable legislation. The methodology used in the production of the present study has departed from the study of the definition of this kind of tourism and its differentiation with other typologies, and the results of this research allowed considering the viability of creation of hotels to receive the tourists, taking into account its peculiarity and the tourist potential.
\end{abstract}

Key words: Tourism; Space; Law; Astronaut.

\footnotetext{
* Diplomada en Turismo. Escuela Politécnica Superior de Gandía. Universitat Politècnica de València. Dirección: Escuela Politécnica Superior de Gandía, C/ Hernán Cortés 11. CEP: 46004 - Valencia (España). Teléfono: 6178773 84. E-mail: anaserranonavalon@gmail.com

** Licenciada y Doctora en Derecho. Profesora Titular de Universidad. Escuela Técnica Superior de Ingeniería Agronómica y del Medio Natural (ETSIAMN). Universitat Politècnica de València. Dirección: Escuela Técnica Superior de Ingeniería Agronómica y del Medio Natural (ETSIAMN). Universitat Politècnica de València. Camino de Vera, s./n. CEP: 46022 - Valencia (España). Teléfono: 646081967. E-mail: frarafer@urb.upv.es
} 


\section{INTRODUCCIÓN}

En el presente trabajo se investiga el concepto de turismo espacial y la comparativa entre el turista "terrestre" y el turista "espacial". También se analizará la legislación vigente espacial y de cómo ésta afecta en sus restricciones a los viajes y viajeros espaciales.

Este estudio intentará despejar algunas dudas que se han creado con esta nueva forma de viajar, como sería el poder considerar o no el turismo espacial como una clase de turismo propiamente dicho o incluso si realmente será posible la creación de hoteles en el espacio para poder acoger a todos estos civiles-turistas espaciales en el caso de que se alargaran sus estancias en el espacio.

\section{EL TURISMO ESPACIAL}

En el presente apartado se va a definir el turismo espacial. Esta definición permitirá conocer los caracteres de este tipo de turismo, para poderlo diferenciar de otros tipos de turismo, ya que participa de algunas características habituales del turismo de aventura. Para ello, es preciso que se remonte a las antiguas civilizaciones y al interés del ser humano por conocer el espacio y los planetas. La literatura científica y literaria de la época hace referencia a viajes imaginarios y a la adoración de los astros (RAMÓN, 2009; SERRANO, 2011).

\subsection{DEFINICIÓN Y CARACTERES}

El deseo del ser humano por viajar más allá de la tierra ha sido una constante en la literatura científica. Se encuentran numerosas referencias en obras que plasman viajes imaginarios a planetas y satélites. Un ejemplo de ello es la obra de Luciano de Samósata, en su Historia Verdadera que describe guerras entre el imperio lunar y solar (CABRERO, 2010). Posteriormente, las referencias literarias a los viajes espaciales han sido reflejadas en la mayoría de los autores que abordaban el género de la ciencia 
ficción. Interesante resulta mencionar también los trabajos emprendidos por Copérnico, Galileo y Newton en los que se inicia el camino para el viaje lunar (RAMÓN, 2009).

No existe ninguna definición de "turismo" universalmente aceptada. Pero, en principio se observa que entre los seres humanos modernos se da el hecho de la búsqueda de periodos de descanso y disfrute distintos al puro hecho de dormir, además de una extraña curiosidad y atracción por desplazarse hacia otros lugares distintos a los de la propia residencia habitual. Esta argumentación parece que es lo único que está bastante claro en relación al turismo.

No resulta sencillo proporcionar una definición de lo que se considera como turismo espacial, ya que, incluso, una definición de turismo resulta compleja. Los elementos que intervienen oscilan entre lo que se considera afluencia de personas, movimiento de éstas, abandono del lugar habitual para trasladarse a otro diferente...

Sin embargo, el turismo, como materia de investigación, comienza a tomar protagonismo en el periodo de entre guerras, entre la Primera (1914-1918) y la Segunda Guerra Mundial (1939-1945) especialmente desde el campo de la economía.

La doctrina especializada ha señalado que fue a través de la aportación de Bormann cuando se introduce en la definición de turismo el concepto de motivación del viaje (MUÑOZ, 2007). Con esta definición se distingue el turismo de lo que es un desplazamiento a un lugar de trabajo, que no constituye turismo propiamente, ya que no se encuentra en ellos el placer o los motivos comerciales u otros en los que sí están presentes en el turismo.

Pero todas las definiciones expuestas hasta aquí olvidan un aspecto fundamental: las relaciones y actividades que se generan en torno a éstos desplazamientos.

Posteriormente, Glucksmann en el año 1935, precisa que el turismo constituye una suma de relaciones entre personas que se desplazan y los naturales del lugar (DACHARY; ARNAIZ, 2006).

Como señala VILORIA (2012), fueron Hunziker y Krapf quienes indicaron que en el turismo se producían relaciones producidas por el desplamiento y permanencia, sin que interviniera una actividad lucrativa principal.

Como indica ESTEBAN (2004, p. 81, n. 2), en la Conferencia Internacional sobre Estadísticas de Viaje y Turismo de la OMT (Organización Mundial del Turismo) 
y que se celebró en 1991, en Otawa (Canadá) se recoge la definición que se acuerda sobre el turismo:

"Las actividades que realizan las personas durante sus viajes y estancias en lugares distintos al de su entorno habitual, por un periodo de tiempo consecutivo inferior a un año, con fines de ocio, por negocio u otros motivos".

Hoy en día existen muchas modalidades del turismo (MARTÍNEZ; RAMÓN, 2011), que es necesario deslindar para poder realizar una definición adecuada del turismo espacial:

- Individual;

- De masas (turismo de sol y playa);

- Cultural (urbano, monumental, arqueológico, funerario, de compras, etnográfico, literario, de formación, gastronómico, enológico, industrial);

- Natural (rural, ecoturismo, agroturismo, ornitológico, ictioturismo, cinegético);

- Activo (parques temáticos, deportivo, aventura, religioso, espiritual, místico, termal o de salud, medico, social, experiencial, itinerante);

- De negocios (reuniones y congresos, seminarios y convenciones, incentivo, fam trips);

- Turismo espacial (relacionado con el espacio y los planetas).

El turismo espacial es una modalidad de turismo en el que se realiza el viaje a más de 100 kilómetros de altura de la Tierra, lo que se considera la frontera del espacio (GARCÍA, s. f.). Se considera como un tipo de turismo derivado del interés del ser humano de conocer el espacio exterior (RAMÓN, 2009). Se incluye un nuevo concepto de viaje, que hasta este momento resultaba impensable, ya que los viajes interespaciales sólo han sido realizados por los astronautas, que cuentan con una preparación específica, pero no por cualquier persona que tenga el sólo interés de conocer lo desconocido. El turismo espacial, por tanto, sería el siguiente paso que daría el ser humano en su conquista del espacio (SERRANO, 2011).

El interés del ser humano en el espacio se ha plasmado a lo largo de los tiempos (SERRANO, 2011). Se materializa cuando los vuelos espaciales tripulados comenzaron a realizarse en la mitad del siglo pasado, un gran número de personas consideró el potencial que suponía el turismo espacial (SERRANO, 2011). Fue Rusia quien propulsó 
el turismo espacial, de un concepto interesante a una realidad, enviando a Dennis Tito, como primer turista espacial del mundo en espacio en el año 2001 (RENGIFO, 2006).

Se denomina turismo espacial a una nueva forma de hacer turismo, a la que se están dedicando muchas importantes empresas privadas, sin ninguna duda, es el aspecto más novedoso en el ámbito del turismo.

Cuando hay que hacer una referencia a los caracteres del turismo espacial lo primero que viene a la mente es el espacio, una nave espacial y la vista de la Tierra a 100 kilómetros de distancia (AA.VV., 2008).

Por el momento sólo se ha llevado a cabo en transportes y estaciones espaciales gubernamentales; pero a principios del siglo XXI las iniciativas privadas han dado los primeros pasos para fabricar vehículos y alojamientos turísticos (MUÑOZ, 2003), produciéndose, en esta variante cósmica, una forma de ostentación de riqueza que ya no se producía con el turismo de masas (MUÑOZ, 2011).

Esta clase de turismo se ha desarrollado en estos momentos, por los avances técnicos y tecnológicos, y por la necesidad de dar un impulso importante, por parte de las empresas privadas, a la carrera espacial emprendida por los gobiernos.

El turismo espacial es cada vez de mayor interés para los ciudadanos civiles, a pesar de que los vuelos suborbitales o incluso orbitales son desde su nacimiento un viaje excesivamente corto para lo que pueden llegar a costar, por muy baratos que se quieran hacer, pero la estancia en el espacio los convertiría en una experiencia única y muy atractiva para el turista en busca de nuevas experiencias.

La iniciativa de las empresas privadas para abrir establecimientos hoteleros es prácticamente una realidad. Citar el caso de Galactic Suite, una empresa catalana que abrirá las puertas de su hotel galáctico en el 2012 (BALLESTEROS, 2012).

\subsection{DIFERENCIACIÓN CON OTROS TIPOS DE TURISMO}

En este punto se analizará cuáles son las principales notas que caracterizan al turismo espacial, y poderlo diferenciar de otros tipos de turismo que pueden mostrar analogías, como es el caso del turismo de aventura, o el turismo de riesgo (SERRANO, 2011). 
Las diferencias entre el turismo terrestre y el turismo espacial son muy claras y evidentes. El turismo terrestre viene efectuado en la Tierra, y el turismo espacial en el espacio, principalmente en la órbita que se encuentra alrededor de la Tierra.

En primer lugar se puede decir que el turismo espacial es un turismo de elite, ya que no se encuentra al alcance de todos, no sólo por el precio de éste, el cual sólo unos pocos privilegiados en el mundo pueden pagarlo, sino también porque hoy en día no todo el mundo puede acceder a esta experiencia.

Para poder experimentar el turismo espacial son necesarias unas condiciones físicas muy buenas y un entrenamiento muy exhaustivo que es proporcionado al turista espacial dentro del precio del viaje y que se realiza entre 3 y 6 meses antes de llevarse a cabo el viaje (SERRANO, 2011).

Otra gran diferencia entre el turismo espacial y el turismo terrestre es que al turismo terrestre está al alcance de todos, ya sea un turismo terrestre de lujo o un turismo terrestre más económico, pero el turismo espacial se realiza en otra atmosfera diferente a la que es habitual, el espacio, y por ello es otra de las evidencias que demuestran que el turismo espacial es sólo para algunos privilegiados.

El turista espacial también se diferencia del turista aventurero terrestre en que el primero se adentra en un terreno desconocido por el turista terrestre, siendo aquél una combinación entre el rol del turista aventurero y del turista de masas (COHEN, 2005).

Se puede definir al turismo espacial como una forma de experimentar sensaciones nuevas, el turista espacial viaja en la nave junto con dos astronautas que hacen posible esta experiencia pero el turista espacial al margen de realizar ciertos experimentos personales lo que realiza mayormente es observar, fotografiar y grabar la grandiosidad de la Tierra desde el espacio (SERRANO, 2011). Sin embargo en el turismo terrestre se pueden experimentar todo tipo de actividades, desde las más arriesgadas hasta las más relajantes.

Dícese turista espacial, aquella persona privilegiada, no astronauta, que viaja al espacio en compañía de expertos del sector (astronautas y cosmonautas). Desembolsando una gran cantidad de dinero para poder efectuar el viaje turístico al espacio que normalmente oscila entre 8 y 10 días (SERRANO, 2011).

El turismo espacial como su definición lo dice es aquel que se realiza a más de 100 kilómetros de la Tierra (GARCÍA, s. f.). En la actualidad no se ve una marcada 
tendencia y no hay viajes turísticos frecuentes al espacio, pero está claramente definido que en un futuro cercano estos viajes formarán parte de los paquetes ofrecidos por distintas agencias (NOZIGLIA, 2011).

El turismo espacial es ya una realidad. La era del turismo en el espacio parecía lejana pero ya hay proyectos muy avanzados que ultiman los preparativos para la gran revolución del turismo (MATEU, 1980).

Nuevos vehículos espaciales que transportarán al turista en vuelos suborbitales se están probando con resultados positivos. Space Adventures, la agencia espacial, afirma que a partir de 2012 podrá enviar a dos turistas a la vez a la Estación Espacial Internacional usando una nave rusa (MORA, 2011). A partir del año 2013 tres turistas al año podrán reserva su pasaje para el viaje, en la compañía de la Estación Espacial en Virginia (SERRANO, 2011).

\subsection{EL VIAJE ESPACIAL Y SUS CARACTERÍSTICAS}

Primero fueron los vuelos de bajo coste y ahora la propuesta más novedosa se centra en los viajes espaciales. Virgin, la empresa del multimillonario británico Richard Branson, ha decidido apostar por el turismo espacial dentro del proceso expansivo de su grupo empresarial, para lo que en 2008 puso en marcha una nueva compañía: Virgin Galactic, especializada en realizar viajes espaciales (FERNÁNDEZ, 2009). Para ello, la compañía filial de Virgin llegó a un acuerdo con la National Aeronautics and Space Administration (en adelante NASA) con el fin de colaborar con los lanzamientos de vuelos al espacio (SERRANO, 2011). La misma autora comenta que de esta forma la compañía británica, realizará la ruta con una dotación de 5 naves espaciales y dos nodrizas. También que las naves están diseñadas para transportar a seis pasajeros y dos pilotos a una altura aproximada de 140 kilómetros en un vuelo suborbital.

Los aspectos que se deben considerar antes de contratar un viaje espacial son los siguientes (SERRANO, 2011): a) Precisión de hacer la reserva con suficiente antelación, generalmente de dos a tres años; b) Posibilidad de que las empresas facilitan el pago aplazado, dado el elevado coste del viaje; c) Necesidad de preparación para realizar el viaje, por lo que se ofertan por parte de las empresas cursillos de aprendizaje; 
d) Obtención de información suficiente en las páginas web de las empresas, en las que facilitan las condiciones y características de cada viaje.

Hay distintas opciones para realizar el viaje de turismo espacial: vuelo espacial orbital, suborbital y la misión Circunlunar DSE-Alpha.

Hay actualmente algunas empresas y agencias especializadas que comercializan actualmente estos viajes espaciales aquí se analizan algunos de ellos:

Space Island (http://www.spaceislandgroup.com/home.html). Esta entidad pretende elaborar una estación espacial y una flota de 6 transbordadores, llevar pasajeros en viajes de una semana, por un coste menor a los 2 millones de euros.

Virgin Galactic (http://www.virgingalactic.com/). La última en lanzarse al mercado turístico. Busca alternativas para ofrecer viajes más económicos, su idea es desarrollar de un cohete reutilizable para que diez personas puedan pasar en el espacio diez días en el 'Hotel Virgin'.

Space Adventures (http://www.spaceadventures.com/). Ofrece entre sus alternativas:

a) Experience Zero-Gravity, se basa en un vuelo de gravedad cero, a través de un avión que describe un arco parabólico creando un efecto simulador de ingravidez. Todas las propuestas estudian la posibilidad de participar en el programa denominado "Pasos al Espacio", lo que da la oportunidad de ir acumulando créditos que luego se descontaran del precio del futuro vuelo espacial.

b) El Borde del Espacio, 25.400 euros. Vuela a 2 1/2 la velocidad del sonido y asciende a altitudes que triplican al Everest. Incluye alojamiento en un hotel de lujo y DVD personalizado.

c) Entrenamiento a Alto G, 19.000 euros, entrenamiento en una aeronave tipo 'L39'. El paquete incluye alojamiento en un hotel de lujo y DVD personalizado.

d) Gravedad Cero en Estados Unidos, 2.900 euros y se trata de prepararse en los Estados Unidos para vivir en el espacio igual que lo hacen los astronautas profesionales.

Una explicación más detallada de las distintas opciones se encuentra en la citada web: http://www.spaceadventures.com/ 


\subsection{APLICACIÓN DEL DERECHO ESPACIAL}

La necesidad de intervención del Derecho en el ámbito espacial nace de la conveniencia de regular las condiciones y relaciones que se hicieron manifiestas con la evolución del hombre, lo que derivó en la carrera espacial, y en el desarrollo de las telecomunicaciones vía satélite (RAMÓN, 2009).

Para poder desarrollar la aplicación del Derecho Espacial es preciso remontar sus antecedentes hasta el pasado y comentar los mismos.

El mayor sueño del hombre desde la antigüedad hasta el día de hoy ha sido soñar con el espacio, intentar averiguar qué había en el, cómo llegar hasta él, entre otros. Este sueño ha pasado de la imaginación de los seres humanos hasta la realidad, debiendo tener en cuenta la justicia y el Derecho para poder regularlo.

Todo se remonta hasta las antiguas civilizaciones donde se consideraba el cielo como una fuente de poder.

Cuando se hace referencia al Derecho Espacial, no sólo hay que referirse al lanzamiento de astronautas o turistas espaciales en cohetes, sino que existen muchos otros temas a tratar. Una vez que se conoce el medio espacial en el entorno de la Tierra, es fácil darse cuenta de que en él hay una serie de condiciones y parámetros que lo convierten en un laboratorio único con cuyo uso se puede mejorar la calidad de la vida de la humanidad. El hecho de que el espacio sea una ventana de observación global de la Tierra y su atmósfera ha permitido la realización de los programas de satélites meteorológicos, de satélites de teledetección para mantener en permanente observación los entornos naturales y la atmosfera, y de los satélites de reconocimiento que han evitado guerras y holocaustos nucleares (RIPOLL, 2008).

Los satélites de comunicaciones y de difusión (TV, radio) que han permitido comunicaciones a escala global, son posibles gracias a que el espacio es también una ventana de escucha única para toda la humanidad.

La microgravedad ha permitido fabricar en el espacio productos esenciales tales como vacunas, catalizadores químicos, aleaciones ligeras y elementos mecánicos y electrónicos que requieran extremada limpieza y contribuyan a mejorar la calidad de vida. 
Otro uso ha contribuido también al mantenimiento de la paz mundial y que es la actividad espacial que más satélites ha lanzado al espacio: los satélites de reconocimiento y vigilancia vulgarmente conocidos como: "satélites espías".

Han proliferado los satélites de comunicaciones que representan la mayor actividad económica y comercial del espacio.

Actualmente, hay compañías internacionales como "Intelsat" que tiene más de 50 satélites en órbita geoestacionaria.

El Derecho espacial no es un nuevo Derecho, es una rama que surge de la necesidad de regular las condiciones y relaciones que se hicieron manifiestas con la evolución del hombre, lo que derivó en la carrera espacial, y en un gran desarrollo de las telecomunicaciones vía satélite. Se entiende por espacio concebido como el cielo atmosférico. El espacio es infinito, indefinido, ilimitado, incorpóreo, abstracto y no concreto. Es un ente, es la nada, es el vacío. Sin embargo el espacio es algo que existe rodeando la superficie de los diferentes Estados que integran el planeta. El Derecho Espacial es una ciencia que busca la regulación de conductas humanas en cualquier ámbito. El Derecho espacial debería ser un factor de desarrollo para todos los países, sobre todo en relación con aquellas actividades espaciales imprescindibles para cualquier proyecto de desarrollo nacional (AA.VV., s. f.).

Por ahora, el Derecho Espacial rige las relaciones jurídicas que se establecen en la Tierra o fuera de ella entre terrícolas, pero si se llegara a conocer a otros, el Derecho debería modificarse y ser revisado desde su base para considerar relaciones jurídicas absolutamente novedosas entre las partes que intervienen (MOSQUERA, 2011).

Cabe destacar que la vida en la estación espacial dará lugar a un sistema de mini sociedad y deberá regirse por el Derecho. Habrá que delimitar la jurisdicción aplicable dado que el conjunto de módulos de diferentes Estados da lugar a la presencia de diferentes Estados de matriculación. De acuerdo con el art. VIII del Tratado sobre los principios que deben regir las actividades de los Estados en la exploración y utilización del espacio ultraterrestre, incluso la Luna y otros cuerpos celestes, conocido como Tratado del Espacio o Carta Magna del Espacio de 1967, es el Estado de matriculación el que conserva la jurisdicción y control sobre el objeto espacial. Sería un modelo seguro de controlar la responsabilidad de los Estados como consecuencia de dichas actividades espaciales. Por vía del acuerdo internacional, los Estados participantes en 
una estación espacial podrían alcanzar un acuerdo diferente que depende de la autonomía de la voluntad de los Estados.

La Carta Magna del Espacio de 1967 junto con el papel de la Organización Naciones Unidas (en adelante ONU) y el ordenamiento jurídico del espacio es lo que rige el espacio exterior. Desde que el globo de los hermanos Montgolfier despegó del campo de Marte de París (CAVALCANTI, PEREIRA y TAVARES, 2006), provocando su caída minutos después, resultaba claro que el Derecho, la norma jurídica también tenía que iniciar un despegue y adaptación a las nuevas tecnologías.

A la industria aeroespacial ha conseguido, a partir de la puesta en órbita del primer satélite artificial, un proceso de exploración, científico y comercial. Las Naciones Unidas fueron el primer foro de debate. En este foro se aplica un criterio de libertad, de no apropiación, de utilización en beneficio de la humanidad lo que condicionan la razón de ser del Derecho Espacial. Se considera que sería mejor denominarlo Derecho de las Actividades Espaciales (GÓMEZ, 2004).

En este punto de la exposición es conveniente indicar que el cuerpo normativo del Derecho Espacial lo integran la siguiente legislación:

1. Tratado sobre los principios que deben regir las actividades de los Estados en la exploración y utilización del espacio ultraterrestre, incluso la Luna y otros cuerpos celestes. Resolución 2222 (XXI) de la Asamblea General, anexo, aprobado el 19 de diciembre de 1966, abierto a la firma el 27 de enero de 1967, entró en vigor el 10 de octubre de 1967.

2. Acuerdo sobre el salvamento y la devolución de astronautas y la restitución de objetos lanzados al espacio ultraterrestre. Resolución 2345 (XXII) de la Asamblea General, anexo, aprobado el 19 de diciembre de 1967, abierto a la firma el 22 de abril de 1968, entró en vigor el 3 de diciembre de 1968.

3. Convenio sobre la responsabilidad internacional por daños causados por objetos espaciales. Resolución 2777 (XXVI) de la Asamblea General, anexo, aprobado el 29 de noviembre de 1971, abierto a la firma el 29 de marzo de 1972, entró en vigor el 11 de septiembre de 1972.

4. Convenio sobre el registro de objetos lanzados al espacio ultraterrestre. Resolución 3235 de la Asamblea General, anexo, aprobado el 12 de noviembre de 1974, abierto a la firma el 14 de enero de 1975, entró en vigor el 15 de septiembre de 1976. 
5. Acuerdo que debe regir las actividades de los Estados en la Luna y otros cuerpos celestes. Resolución 34/68 de la Asamblea General, anexo, aprobado el 5 de diciembre de 1979, abierto a la firma el 18 de diciembre de 1979, entró en vigor el 11 de julio de 1984 .

Del mismo modo, añadir la formulación y aprobación de distintas resoluciones de la Asamblea General de Naciones Unidas que afectan al espacio:

1. Declaración de los principios jurídicos que deben regir las actividades de los Estados en la exploración y utilización del espacio ultraterrestre, aprobada el 13 de diciembre de 1963. Resolución 1962 (XVII) de la Asamblea General.

2. Principios que han de regir la utilización por los Estados de satélites artificiales de la Tierra para las transmisiones internacionales directas por televisión, aprobados el 10 de diciembre de 1982. Resolución 37/92 de la Asamblea General.

3. Principios relativos a la teleobservación de la Tierra desde el espacio, aprobados el 3 de diciembre de 1986. Resolución 41/65 de la Asamblea General.

4. Principios pertinentes a la utilización de fuentes de energía nuclear en el espacio ultraterrestre, aprobados el 14 de diciembre de 1992. Resolución 47/68 de la Asamblea General.

5. Declaración sobre la cooperación internacional en la exploración y utilización del espacio ultraterrestre en beneficio e interés de todos los Estados, teniendo especialmente en cuenta las necesidades de los países en desarrollo, aprobada el 13 de diciembre de 1996. Resolución 51/122 de la Asamblea General.

\subsection{PROBLEMAS JURÍDICOS QUE PLANTEA EL TURISMO ESPACIAL: CONSIDERACIÓN SOBRE SI ES UNA CLASE DE TURISMO}

Si se considera al turista espacial como astronauta o más correctamente como personal o tripulación de una nave espacial, será necesario analizar qué normas le son aplicable y en qué medida (Más ampliamente, véase, ALEMÁN, 1962; ESTRADE, 1964; BRAVO, 2005; RAMÓN, 2009).

El hecho de que el astronauta pueda ser considerado como "enviado de la Humanidad" no significa que goce de inmunidad diplomática. El astronauta sigue siendo nacional de su Estado, ya que no se ha establecido ninguna nacional cósmica. 
La Carta Magna Espacial o Tratado del Espacio está constituido por un preámbulo y 17 artículos. El más polémico es el art. 2 que declara al espacio exterior patrimonio común de la humanidad, no susceptible de aprobación. También el art. 4 que prohíbe la militarización del espacio, reservándolo sólo para actividades pacíficas. En la parte declarativa del Tratado se anuncia los ideales que guían el Tratado como que las actividades espaciales sean para el beneficio de la humanidad "en bien de todos los pueblos, sea cual fuere su grado de desarrollo económico y científico". Explicita los principios de libertad y de igualdad. Prohíbe la apropiación o reivindicación de soberanía. Exige el fomento de la cooperación y la comprensión internacionales. Prohíbe la colocación de objetos portadores de armas nucleares o de destrucción masiva y prescribe que la Luna y los cuerpos celestres se utilizarán exclusivamente con fines pacíficos (Véase más ampliamente, OROZCO, 2012).

A continuación cabe destacar la doctrina ha indicado la necesidad de elaboración de un Código de Conducta para ser aplicado a la Estación Espacial Internacional (GONZÁLEZ, 2007).

En este sentido, hay que indicar la regulación del Acuerdo entre el Gobierno de Canadá, los Gobiernos de los Estados miembros de la Agencia Espacial Europea, el Gobierno de Japón, el Gobierno de la Federación de Rusia y el Gobierno de los Estados Unidos de América relativo a la cooperación sobre la Estación Espacial Civil Internacional, y Acuerdo relativo a la aplicación del Acuerdo Intergubernamental sobre la Estación Espacial hasta su entrada en vigor, hechos en Washington el 29 de enero de 1998 (Boletín Oficial del Estado núm. 5, de 6 de enero de 1999).

En base a los artículos anteriores de este Código de conducta se plantea la siguiente pregunta: ¿la estancia o envío de un turista espacial se encontraría dentro de esas actividades? Hay que indicar que la respuesta es positiva, ya que en la Estación Espacial se pueden realizar estancias por parte de personal visitante, con lo que entraría dentro del marco de las actividades realizadas.

Otra cuestión a tener en cuenta es la exoneración de responsabilidad en el caso del turista espacial. Teniendo en cuenta que puede firmar una cláusula en la que exonere de responsabilidad al Estado, y que asuma todos los riesgos en el caso de daños causados en el espacio. Ello se encuadra en lo indicado en el Convenio sobre la 
responsabilidad internacional por daños causados por objetos espaciales, mencionado anteriormente.

El Estado que patrocine el viaje del turista no podría ser demandado por otro Estado parte, ni el turista en este caso podría demandar a otro Estado parte por los daños sufridos. He aquí la conclusión de que los turistas espaciales se regirán con las mismas leyes que los astronautas y deberán de respetarlas con si se trataran de las Leyes del planeta Tierra ya que tienen igual validez.

\section{TIPOLOGÍA DE TURISTAS Y CONSUMIDORES DE TURISMO ESPACIAL}

En el presente apartado se va a analizar qué tipo de turista puede estar interesado en el turismo espacial. Hay que, desde luego, distinguir el turista espacial como turista y el astronauta como profesional. En el caso del turista espacial se tiene que tener em cuenta que puede ser una persona sin formación profesional de astronauta; sin embargo, el astronauta no puede ser considerado como turista, al realizar su trabajo como un profesional, con una formación específica (RAMÓN, 2009).

Sin embargo se define al astronauta como el profesional que lleva a cabo su trabajo en el espacio ultraterrestre, que tripula una astronave o que está entrenado para este trabajo (SERRANO, 2011).

Se reconoce como astronauta a todo aquel que viaje en una nave espacial y a toda persona seleccionada para un viaje espacial. Nada se opone a conceder a los pasajeros el mismo estatuto que al personal de la nave espacial y de aquí nacería el siguiente planteamiento: ¿el turista espacial podría ser considerado como un astronauta?. Esta pregunta se puede platear desde muchos aspectos, entre otros, el económico, el legislativo o incluso en el aspecto social para poder plantear correctamente las diferencias entre unos y otros.

En cuanto al aspecto económico, nace ya una gran diferencia entre unos y otros, ya que el turista espacial debe desembolsar grandes sumas de dinero para poder permitirse el lujo de viajar al espacio y el astronauta cobra un sueldo ya que bajo su responsabilidad se encuentra el turista espacial. 
Para los astronautas dejar la Tierra y viajar al espacio significa Meses de entrenamiento, bajón emocional, preparación psicológica para cualquier adversidad, aislamiento del resto del mundo, falta de reconocimiento económico, emociones fuertes, miedo, entre otros aspectos. El viaje al espacio es una experiencia tan cargada de adrenalina como de temores. $\mathrm{Y}$ sin embargo se entiende que por parte del turista espacial sus sentimientos son más de adrenalina y pasión por descubrir algo nuevo, una experiencia única, un viaje a lo desconocido.

Muchas de las preguntas que se pueden formular al hablar sobre el turismo espacial son todas aquellas que se podrían plantear desde la parte del astronauta, ya que hay que tener en cuenta que es su profesión y no se tiene en cuenta todo lo que a ellos les afecta o repercute. Se conoce por parte de la sociedad cuál es la misión de un astronauta cuando deja este planeta, ¿pero cómo vive una experiencia por la que pocas personas en el mundo han pasado? ¿Cuál es la carga emocional y económica para su familia? ¿Sufren los astronautas secuelas físicas o psicológicas después de las misiones espaciales? ¿Reciben sueldos millonarios?

Al regresar a la Tierra, muchos tardan semanas en recuperarse. La labor que desarrollaron en el espacio es hasta tal punto estresante que volver a casa trae consigo una sensación de relajación que puede llegar a desembocar en una enfermedad.

Durante el viaje espacial, estos científicos son más que nunca dueños de cada uno de sus movimientos y responsables de las personas que llevan a su cargo. Si algo sale mal, desde aquí abajo sólo pueden recibir consejos; las decisiones finales son exclusivamente de ellos.

En cuanto a la legislación actualmente no existe ningún tipo de concreto para los turistas espaciales, con lo que se les aplica la misma legislación que todo astronauta debe respetar y cumplir en su estancia en el espacio.

\section{LOS HOTELES ESPACIALES}

Se va a analizar en este punto cuál es el panorama actual respecto a la creación y posible implantación de los denominados hoteles espaciales, con la finalidad de acoger a los turistas espaciales. 
Dentro de este punto es conveniente analizar dos tipos de comercialización en el espacio, presente o futura. En primer lugar se habla de futuros hoteles en el espacio o en la Luna y en segundo lugar comentar la venta ilegal de parcelas lunares que se pueden adquirir en algunas webs.

Con toda seguridad llegará un día donde el lujo y el hedonismo terrenal quedarán atrás y el planeta será un referente giratorio de quien pasa vacaciones en una sofisticada nave espacial camino de Marte, viendo desde la ventana telemétrica de su camarote a sus trabajadores en la Tierra.

Existen proyectos de desarrollo de hoteles espaciales que servirán de apoyo para los futuros vuelos turísticos orbitales. Se está desarrollando y experimentando con modelos de hoteles espaciales que permanecerán en órbita alrededor de la Tierra (SOLER, 2010). Se habla de hoteles en el espacio (SOMALO, 2007).

Uno de los principales proyectos de hoteles espaciales es el creado por Galactic Suite Spaceresort (galacticsuitespaceresort.com/) diseña y promueve el primer hotel espacial, el segmento orbital de la GALACTIC SUITE EXPERIENCE, una mini estación espacial orbitando en órbita terrestre baja (LEO) dedicada a acomodar pasajeros privados, los turistas espaciales. Está concebida como un refugio no permanente para tripulación y turistas.

Como ha indicado la European Aeronautic Defence and Space Company (en adelante EADS) Astrium, la arquitectura de la estación se define utilizando módulos modificados a partir de la tecnología del Vehículo Automatizado de Transferencia (en adelante ATV), lo que sustentaría los turistas espaciales para estancias cortas, de 4 a 6 días (ESA, 2008). La primera fase de la estación establece un único módulo orbital como free flyer, considerado como el primer ladrillo de una estación orbital autónoma El objetivo final de la compañía es desarrollar la primera cadena hotelera espacial en el mundo con hábitats espaciales modulares y hacer el turismo espacial accesible al público.

La empresa calcula que en el mundo hay más de 40.000 personas que pueden pagar esta cantidad por lo que esperan recibir reservas suficientes para que el proyecto sea un éxito.

También se especula sobre posibles hoteles establecidos en el suelo lunar aproximadamente en el año 2030 (AA.VV., 2009). Expertos en arquitectura 
extraterrestre trabajan en diferentes proyectos relacionados con el turismo espacial para lograr este fin. Las primeras experiencias obtenidas con los hoteles orbitales marcarán tendencias para las futuras instalaciones lunares. Cualquier arquitectura espacial debe estar preparada para soportar las condiciones extremas de los ambientes donde serán emplazadas. En el caso de la luna, los días y las noches durarán 14 días terrestres. Las estructuras deben soportar lluvias de meteoritos y resolver el problema de la falta de aire y agua. Por otra parte, el hecho de que la gravedad lunar sea seis veces inferior a la terrestre abre nuevas posibilidades arquitectónicas que permitirán crear edificios con conceptos y diseños innovadores imposibles de construir en la Tierra.

Entre los proyectos más conocidos de alojamiento lunar destacan el Lunatic, un hotel proyectado por el arquitecto holandés Hans Jurgen Rombaut y un nuevo hotel Hilton de 5000 habitaciones (http://www.rombaut.nl/). Ambos se construirían en el suelo lunar.

Otros proyectos relacionados con el turismo espacial se basan en hoteles situados en la órbita de la luna, como el propuesto por la constructora japonesa Shimizu (http://www.shimz.co.jp/english/).

A los pocos años de la puesta en marcha de su primera línea espacial, Virgin Galactic tiene previsto bajar los precios hasta cifras cercanas a los 20.000 dólares por trayecto (http://www.virgingalactic.com/). Se convertirá entonces en un viaje al alcance de millones de personas en todo el mundo, consolidando el turismo espacial como una fuente de negocio rentable que impulsará nuevos desarrollos tecnológicos destinados a la exploración espacial. Probablemente entonces se harán más asequibles los vuelos orbitales, con una duración de varias horas o incluyo días.

La era del turismo espacial ya ha comenzado. Quizá dentro de 50 o 100 años sea uno de los sectores que más ingresos genere. Aún falta mucho por hacer. La regulación de las leyes y competencias que regirán en el espacio exterior tendrán que debatirse y habrá que afrontar muchos retos técnicos. Lo que es innegable es que el turismo espacial ya está en marcha.

En cuanto a la venta de parcelas lunares antes de nada resulta interesante hacer referencia a esta página web: www.lunarembassy.com se trata de la página web que se encarga de las parcelas lunares y de los diferentes planetas. 
Si bien el Tratado del Espacio Exterior establecido por las Naciones Unidas en 1967 especifica que ningún Estado ni Nación puede apropiarse de un planeta o una estrella, el mismo no hace referencia a particulares, por los cual Dennis Hope en 1980 registró a su nombre la Luna y todos los planetas del Sistema Solar, poniendo a la venta terrenos en el satélite natural de la Tierra y el planeta Marte (LISTNER, 2003).

Actualmente Denis Hope comercializa 1500 terrenos por día, de media hectárea de la Luna, Mercurio, Venus, y Marte y su compañía vendió terrenos lunares a 6 millones de personas en 80 países a más de 1300 corporaciones (http://www.moonshop.com/).

\section{CONCLUSIONES}

Partiendo de la base de que el turismo espacial tiene un elevado coste económico, se puede decir que es un turismo de elite, sólo para unos pocos privilegiados en el mundo. Las compañías que promocionan este tipo de turismo, están intentando por todos los medios abaratar sus costes para que en un futuro (se habla del año 2030-2050) el turismo espacial, ya sean los viajes orbitales de unas horas de duración o las estancias en los futuros hoteles espaciales, estén al alcance de todos.

Cuando se habla en prensa, en televisión o los mismos científicos, de que esta clase de turismo estará al alcance de todos, realmente es una quimera, ya que aún faltaría mucho tiempo para ellos y su precio debería disminuir en un $90 \%$ su coste actual.

Cuando a lo largo de este estudio se plantea la pregunta de si el turista espacial debería ser considerado un astronauta, la respuesta es clara: No. Cuando se habla de los aspectos legales es preciso decir que debido a un "vacío legal" si que, en muchos aspectos, se le aplican (a los turistas espaciales) la gran mayoría de las leyes existentes vinculadas a los astronautas, pero al no existir unas Leyes concretas o un Tratado que rija al turista espacial, concretamente, se le aplican las existentes.

También dentro de esta diferencia entre el astronauta y el turista espacial hay que ser justos, y no considerar al turista como un astronauta debido a que la preparación física, psíquica y los estudios no son los mismos. Un astronauta es una persona que ha 
estudiado por ello y le pagan por realizar su trabajo y el turista espacial, a día de hoy, es una persona que desembolsa una gran cantidad de dinero por viajar al espacio. Es decir el turista viaja por placer y el astronauta viaja para realizar su trabajo.

Para concluir este trabajo es interesante plantear la siguiente pregunta: turismo espacial, ¿sueño o realidad? Personalmente a la conclusión de este articulo, se considera que es toda una realidad. Y sí, se tardará aún mucho tiempo para que se encuentre al alcance de todos, de la sociedad, pero actualmente es una realidad que evoluciona a pasos agigantados.

\section{REFERENCIAS}

AA.VV. Derecho espacial y telecomunicaciones. Disponível em: <http://www.tuobra.unam.mx/publicadas/020428174637-DERECHO.html>. Acesso em: 09/04/2013.

Estudios de Derecho aeronáutico y espacial. XXXI JORNADAS LATINOAMERICANAS DE DERECHO AERONÁUTICO Y ESPACIAL (Sevilla, España, 20-23 de marzo de 2007), Mario Folchi, Mª Jesús Guerrero Lebrón y Agustín Madrid Parra (Coordinadores). Madrid: Marcial Pons, 2008.

Turismo espacial. Maquinalia, n. 1, 2009. Disponível em: <http://maquinalia.com/turismo-espacial.html>. Acesso em: 09/04/2013.

ACUERDO SOBRE EL SALVAMENTO Y LA DEVOLUCIÓN DE ASTRONAUTAS Y LA RESTITUCIÓN DE OBJETOS LANZADOS AL ESPACIO ULTRATERRESTRE. Resolución 2345 (XXII) de la Asamblea General, anexo, aprobado el 19 de diciembre de 1967, abierto a la firma el 22 de abril de 1968, entró en vigor el 3 de diciembre de 1968. Disponível em: <http://www.oosa.unvienna.org/pdf/publications/STSPACE11S.pdf $>$. Acesso em: 09/04/2013.

ACUERDO QUE DEBE REGIR LAS ACTIVIDADES DE LOS ESTADOS EN LA LUNA Y OTROS CUERPOS CELESTES. Resolución 34/68 de la Asamblea General, anexo, aprobado el 5 de diciembre de 1979, abierto a la firma el 18 de diciembre de 1979, entró en vigor el 11 de julio de 1984. Disponível em: <http://www.oosa.unvienna.org/pdf/publications/STSPACE11S.pdf >. Acesso em: 09/04/2013. 
ACUERDO ENTRE EL GOBIERNO DE CANADÁ, LOS GOBIERNOS DE LOS ESTADOS MIEMBROS DE LA AGENCIA ESPACIAL EUROPEA, EL GOBIERNO DE JAPÓN, EL GOBIERNO DE LA FEDERACIÓN DE RUSIA Y EL GOBIERNO DE LOS ESTADOS UNIDOS DE AMÉRICA RELATIVO A LA COOPERACIÓN SOBRE LA ESTACIÓN ESPACIAL CIVIL INTERNACIONAL, Y ACUERDO RELATIVO A LA APLICACIÓN DEL ACUERDO INTERGUBERNAMENTAL SOBRE LA ESTACIÓN ESPACIAL HASTA SU ENTRADA EN VIGOR, hechos en Washington el 29 de enero de 1998 (Boletín Oficial del Estado núm. 5, de 6 de enero de 1999). Disponível em: <http://www.boe.es/diario_boe/txt.php?id=BOE-A-1999-215>. Acesso em: 09/04/2013.

ALEMÁN VELASCO, M. Los secretos y las leyes del espacio. México: Editorial Helio-México, 1962.

BALleSteros, F. Viure en l'espai. Mètode, 2012, p. 124-125. Disponível em: <http://130.206.88.107/revistes224/index.php/Metode/article/viewFile/64807/64676>. Acesso em: 09/04/2013.

BRAVO NAVARRO, M. Diccionario de cuestiones jurídicas aéreo-espaciales. Madrid, Editorial Reus, S.A., 2005.

CABRERO, M. del C. El Icaormenipo de "Luciano de Samóstata": la risa, entre el spoudogeloîon y la carnavalesca de Bajtin. Cuadernos de filología clásica: Estudios griegos e indoeuropeos, n. 20, 2010, p. 215-230.

CAVALCANTI SOARES, L. A.; PEREIRA DE MACEDO, A. K.; TAVARES VIANA, L. J. La política de costo low-cost, low fare. Turiciencia. PRIMER CONGRESO DE CIENCIAS APLICADAS AL TURISMO, 2006, p. 1-4. Disponível em: <http://www.ensayoes.com/tw_files/1912/d-1911620/7z-docs/7.pdf>. Acesso em: 09/04/2013.

COHEN, E. Principales tendencias en el turismo contemporáneo. Política y Sociedad, v. 42, n. 1, 2005. Disponível em:

<http://revistas.ucm.es/index.php/POSO/article/view/24128>. Acesso em: 08/04/2013.

CONVENIO SOBRE LA RESPONSABILIDAD INTERNACIONAL POR DAÑOS CAUSADOS POR OBJETOS ESPACIALES. Resolución 2777 (XXVI) de la Asamblea General, anexo, aprobado el 29 de noviembre de 1971, abierto a la firma el 29 de marzo de 1972, entró en vigor el 11 de septiembre de 1972. Disponível em: <http://www.oosa.unvienna.org/pdf/publications/STSPACE11S.pdf>. Acesso em: 09/04/2013. 
CONVENIO SOBRE EL REGISTRO DE OBJETOS LANZADOS AL ESPACIO ULTRATERRESTRE. Resolución 3235 de la Asamblea General, anexo, aprobado el 12 de noviembre de 1974, abierto a la firma el 14 de enero de 1975, entró en vigor el 15 de septiembre de 1976. Disponível em:

<http://www.oosa.unvienna.org/pdf/publications/STSPACE11S.pdf>. Acesso em: 09/04/2013.

DACHARY, A. C.; ARNAIZ BURNE, S. M. El estudio del turismo ¿Un paradigma en formación?. Estudios y perspectivas en turismo, v. 16, n. 2, abril-junio 2006. Disponível em: <http://www.scielo.org.ar/scielo.php?pid=S185117322006000200004\&script=sci_arttext $>$. Acesso em: 08/04/2013.

DECLARACIÓN DE LOS PRINCIPIOS JURÍDICOS QUE DEBEN REGIR LAS ACTIVIDADES DE LOS ESTADOS EN LA EXPLORACIÓN Y UTILIZACIÓN DEL ESPACIO ULTRATERRESTRE, aprobada el 13 de diciembre de 1963. Resolución 1962 (XVII) de la Asamblea General. Disponível em: <http://www.oosa.unvienna.org/pdf/publications/STSPACE11S.pdf>. Acesso em: 09/04/2013.

DECLARACIÓN SOBRE LA COOPERACIÓN INTERNACIONAL EN LA EXPLORACIÓN Y UTILIZACIÓN DEL ESPACIO ULTRATERRESTRE EN BENEFICIO E INTERÉS DE TODOS LOS ESTADOS, TENIENDO ESPECIALMENTE EN CUENTA LAS NECESIDADES DE LOS PAÍSES EN DESARROLLO, aprobada el 13 de diciembre de 1996. Resolución 51/122 de la Asamblea General. Disponível em:

<http://www.oosa.unvienna.org/pdf/publications/STSPACE11S.pdf>. Acesso em: 09/04/2013.

ESA. Vehículo automatizado de Transferencia (ATV), 2008. Disponível em: $<$ http://esamultimedia.esa.int/docs/ATV/infokit/spanish/JulesVerneATV_Info_Kit_es.p df>. Acesso em: 09/04/2013.

ESTEBAN TALAYA, A. Modelos de la demanda turística en España: segmentación por países de procedencia, Mediterráneo Económico: "Las nuevas formas de turismo". Almería: Caja Rural Intermediterránea Cajamar, 2004. Disponível em: $<$ http://www.publicacionescajamar.es/pdf/publicaciones-periodicas/mediterraneoeconomico/5/5-56.pdf>. Acesso em: 08/04/2013.

ESTRADE RODOREDA, S. EI Derecho ante la conquista del espacio. Barcelona: Areil, 1964. 
FERNÁNDEZ ARRIBAS, G. El turista espacial y su consideración en el Derecho Internacional público. Revista Brasileira de Direito Aeronáutico e Espacial, diciembre 2009. p. 1-18. Disponível em:

<http://www.sbda.org.br/revista/Anterior/1811.pdf>. Acesso em: 09/04/2013.

GALACTIC SUITE. Disponível em: <galacticsuitespaceresort.com/>. Acesso em: 09/04/2013.

GARCÍA, M. Turismo espacial: innovación comercial. XVIII SEMINARIO DE ADMINISTRACIÓN PÚBLICA DESAFÍOS INTERNACIONALES DE LA GESTIÓN PÚBLICA, s. f., p. 85. Disponível em:

<http://www.ordenjuridico.gob.mx/Publicaciones/CDs2010/CDEspacio/pdf/OP18.pdf>. Acesso em: 08/04/2013.

GÓMEZ ROJO, M. E. Introducción al Derecho aéreo y espacial. Su enseñanza en Francia ante la implantación del sistema europeo de educación superior. Disponível em: <http://www.estig.ipbeja.pt/ ac_direito/gomezrojo2004a.pdf>. Acesso em: 09/04/2013.

GONZÁLEZ FERREIRO, E. C. La estación espacial internacional. Régimen jurídico. Madrid: Instituto Iberoamericano de Derecho aeronáutico y del espacio, 2007.

LISTNER, M. Ownership and Exploitation of Outer Space: A look at Foundational Law and Future Legal Challenges to Current Claims. The Regent J Int'l L, v. 1, 2003, p. 75. Disponível em:

$<$ http://heinonline.org/HOL/LandingPage?collection=journals\&handle=hein.journals/re gjill\&div=10\&id=\&page=> . Acesso em: 09/04/2013.

LUNAR ARCHITECTURE. Disponível em: <http://www.rombaut.nl/>. Acesso em: 09/04/2013.

LUNAR EMBASSY. Disponível em: <http://www.lunarembassy.com>. Acesso em: 09/04/2013.

MARTÍNEZ ACEBES, A. S.; RAMÓN FERNÁNDEZ, F. Estudio comparativo de empresas de turismo de aventura de la provincia de Valencia. Gran Tour: Revista de Investigaciones Turísticas, n. 3, 2011, p. 51-68. Disponível em: <http://www.eutm.es/revista/numero3/pdf/Articulo3.pdf>. Acesso em: 08/04/2013.

MATEU SANCHO, P. Los viajes espaciales. Barcelona: Salvat Editores, S.A., 1980.

MOON SHOP. Disponível em: <http://www.moonshop.com/>. Acesso em: 09/04/2013. 
MORA, H. El turismo espacial. Héctormora.tv, 2011. Disponível em: $<$ http://www.hectormora.tv/index.php?option=com_content $\&$ view $=$ article \&id=127\%3A penalosa-de-uribe $\&$ catid $=40 \% 3$ Atips-para-viajeros $\&$ Itemid=179>.$\quad$ Acesso $\mathrm{em}$ : 09/04/2013.

MOSQUERA AGUIRRE, G. El Derecho Espacial: un desafío para Ecuador. Gaceta Jurídica. Coordinadora Andina de los Derechos Humanos, 2011, p. 1-26.

Disponível em:

<http://www.cadhu.com.ec/Cadhu\%20Public\%20Ultima\%2011\%20Julio\%202011/Abg $\%$ 20Soc\%20Gabriela\%20Catalina\%20Mosquera\%20Aguirre/1\%20Abg.\%20Soc.\%20G abriela\%20Catalina\%20Mosquera\%20Aguirre.pdf>. Acesso em: 09/04/2013.

MUÑOZ DE ESCALONA, F. El turismo explicado con claridad, 2007. Disponível em:

$<$ http://ciam.ucol.mx/directorios/1153/Turismo\%20sis\%20admvos/El\%20Turismo\%20 Explicado\%20Con\%20Claridad.PDF>. Acesso em: 14/07/2013.

. Los mitos del turismo (con nuevas remociones de sus estancadas aguas).

Turydes, v. 4, n. 11, 2011. Disponível em:

<http://www.eumed.net/rev/turydes/11/fme2.html>. Acesso em: 14/07/2012.

NOZIGLIA, F. El turismo espacial y sus pioneros. Extremisimo: blog de tecnología, 2011. Disponível em: <http://extremisimo.com/el-turismo-espacial-y-sus-pioneros/>. Acceso em: 09/04/2013.

OROZCO SÁENZ, M. Cuestiones jurídicas relacionadas con el estatuto jurídico del astronauta en el marco de las Naciones Unidas y de la Estación Espacial Internacional. Revista electrónica de Estudios Internacionales, n. 23, 2012, p. 1-25. Disponível em: <http://dialnet.unirioja.es/servlet/articulo?codigo=4043525>. Acesso em: 09/04/2013.

PRINCIPIOS QUE HAN DE REGIR LA UTILIZACIÓN POR LOS ESTADOS DE SATÉLITES ARTIFICIALES DE LA TIERRA PARA LAS TRANSMISIONES INTERNACIONALES DIRECTAS POR TELEVISIÓN, aprobados el 10 de diciembre de 1982. Resolución 37/92 de la Asamblea General. Disponível em: <http://www.oosa.unvienna.org/pdf/publications/STSPACE11S.pdf>. Acesso em: 09/04/2013.

PRINCIPIOS RELATIVOS A LA TELEOBSERVACIÓN DE LA TIERRA DESDE EL ESPACIO, aprobados el 3 de diciembre de 1986. Resolución 41/65 de la Asamblea General. Disponível em:

$<$ http://www.oosa.unvienna.org/pdf/publications/STSPACE11S.pdf $>$. Acesso em: 09/04/2013. 
PRINCIPIOS PERTINENTES A LA UTILIZACIÓN DE FUENTES DE ENERGÍA NUCLEAR EN EL ESPACIO ULTRATERRESTRE, aprobados el 14 de diciembre de 1992. Resolución 47/68 de la Asamblea General. Disponível em: <http://www.oosa.unvienna.org/pdf/publications/STSPACE11S.pdf $>$. Acesso em: 09/04/2013.

RAMÓN FERNÁNDEZ, F. Derecho Espacial. Temas 1 a 5, y Temas 6 a 10, Valencia: Servicio de Publicaciones de la Universidad Politècnica de Valencia, 2009.

RENGIFO GALLEGO, J. I. Rutas culturales y turismo en el contexto español. PH 60. Especial Monográfico: Itinerarios y rutas culturales, 2006. Disponível em: <http://www.iaph.es/revistaph/index.php/revistaph/article/view/2263/2263\#.UWNAtmbucw>. Acesso em: 09/04/2013.

RIPOLL MUNTANER, A. Texto-guión de la Conferencia pronunciada por el Académico D. Andrés Ripoll Muntaner con motivo de la "Jornada Espacial" celebrada el 25 de septiembre de 2007. Boletín del Centro Español de Derecho Espacial (CEDE), n. 18, 2008, p. 1-47. Disponível em: <http://www.plataformaaeroespacial.org/noticias/boletin_enero2008-v7.pdf>. Acesso em: 09/04/2013.

SERRANO NAVALÓN, A. I. Aspectos legales y socio-económicos del turismo espacial, Trabajo Final de Carrera de la Diplomatura de Turismo, dirigido por Francisca Ramón Fernández, 2011. Disponível em:

<http://riunet.upv.es/bitstream/handle/10251/12843/PFC\%20Ana\%20Isabel\%20\%20Ser rano\%20Navalon.pdf?sequence=1>. Acesso em: 01/04/2013.

SHIMIZU. Disponível em: <http://www.shimz.co.jp/english/>. Acesso em: 09/04/2013.

SOLER MONTELlANO, A. Colonización espacial. Revista Hipo Tesis, n. 1, 2010, p. 12.

SOMALO, C. Últimas fronteras: hoteles en el espacio y en el fondo del mar. Experimenta: diseño, arquitectura, comunicación, n. 58, 2007, p. 86-90.

SPACE ADVENTURES. Disponível em: <http://www.spaceadventures.com/>. Acesso em: 09/04/2013.

SPACE ISLAND. Disponível em: <http://www.spaceislandgroup.com/home.html>. Acesso em: 09/04/2013.

TRATADO SOBRE LOS PRINCIPIOS QUE DEBEN REGIR LAS ACTIVIDADES DE LOS ESTADOS EN LA EXPLORACIÓN Y UTILIZACIÓN DEL ESPACIO 
ULTRATERRESTRE, INCLUSO LA LUNA Y OTROS CUERPOS CELESTES DE 1967 (Conocido como Tratado del Espacio o Carta Magna del Espacio). Disponível em: <http://www.oosa.unvienna.org/pdf/publications/STSPACE11S.pdf>. Acesso em: 09/04/2013.

VILORIA CEDEÑO, N. E. Desarrollo turístico y su relación con el transporte. Gestión Turística, n. 17, 2012, p. 23-36. Disponível em:

<http://mingaonline.uach.cl/pdf/gestur/n17/art02.pdf>. Acesso em: 08/04/2013.

VIRGIN GALACTIC. Disponível em: <http://www.virgingalactic.com/>. Acesso em: 09/04/2013.

\section{OBSERVACIONES}

El presente trabajo tiene su origen en el Trabajo Final de Carrera de la Diplomatura de Turismo de Ana Isabel Serrano Navalón, titulado: “Aspectos legales y socio-económicos del turismo espacial", dirigido por Francisca Ramón Fernández. Dicho trabajo es accesible en el repositorio institucional de la Universitat Politècnica de València, en:

<http://riunet.upv.es/bitstream/handle/10251/12843/PFC\%20Ana\%20Isabel\%20\%20Ser rano\%20Navalon.pdf? sequence $=1>$.

Sobre la base de ese trabajo se ha elaborado el presente artículo, que no ha sido previamente publicado en ninguna revista científica, y que se incorpora al presente artículo la cita del trabajo previo, a efectos de evitar problemas de publicación.

Recebido em: 29-07-2012 (primeira versão)

Recebido em: 10-04-2103 (segunda versão)

Aceito para publicação em: 10-05-2013 